\title{
EL DEPORTE COMO MECANISMO PROPAGANDÍSTICO DE IDENTIDAD NACIONAL EN ESPAÑA
}

SPORT AS A NATIONAL IDENTITY PROPAGANDA MECHANISM IN SPAIN

O ESPORTE COMO MECANISMO PROPAGANDÍSTICO DE IDENTIDADE NACIONAL NA ESPANHA

María Inés Táboas-Pais*, Inma Canales-Lacruz* , Ana Rey-Cao***, María Jesús Perich-Sancho****

Palabras clave: Formulación de políticas.

Deportes.

Propaganda.

Cultura.

\section{Keywords:}

Policy

making.

Sports.

Propaganda.

Culture.

Palavras chave: Formulação de políticas.

Esportes.

Propaganda.

Cultura.
Resumen: El objetivo de este trabajo fue identificar el uso del deporte como mecanismo propagandístico de identidad nacional en los programas de los partidos políticos que presentaron candidatura a las elecciones generales de 2011 en España. Para ello, se analizó el contenido de estos textos. Los resultados del análisis constataron que ocho programas electorales utilizan el deporte en su discurso programático como estrategia para reforzar la identidad cultural de un territorio y patrocinar la nación. Sobre todo, son los partidos nacionalistas los que más uso hacen de este discurso, circunscribiéndolo al independentismo.

Abstract: This work looks into the use of sport as a mechanism of national identity propaganda in political parties' programs in Spain's 2011 general elections. The study is based on content analysis of those programs. Results indicate that the election programs used sport to reinforce territorial cultural identity and strengthen the idea of nation. Nationalist parties were the ones to use that discourse most often, placing it under within Independentism.

Resumo: O objetivo deste trabalho foi o de identificar o uso do esporte como mecanismo propagandístico de identidade nacional nos programas dos partidos políticos que apresentaram candidaturas nas eleições gerais de 2011 na Espanha. Para isso, foram analisados os conteúdos desses programas. Os resultados da análise constataram que oito programas eleitorais utilizam o esporte em seu discurso programático como estratégia para reforçar a identidade cultural de um território e patrocinar a nação. Sobretudo, são os partidos nacionalistas os que mais fazem uso desse discurso, circunscrevendo-o ao independentismo.
**Universidad Católica San Antonio. Murcia, España.

E-mail: mtaboas@ucam.edu.

**Universidad Zaragoza. Zaragoza, España.

E-mail: bromato@unizar.es

***Universidad de Vigo. Vigo, España.

E-mail: anacao@uvigo.es

****Gobierno de Aragón. Aragón. Departamento de Industria e Innovación. España.

E-mail: mjperich@hotmail.com

Recebido em: 13-01-2015

Aprovado em: 23-07-2015

(c) (i) () Licence 


\section{INTRODUCCIÓN}

Para Hobsbawm (1992) la identidad nacional es una construcción social, un invento contemporáneo. Más concretamente, este autor considera que es producto del liberalismo económico de mediados del siglo XIX para erigir el concepto de nacionalismo en torno a la nación. Se consideraban tres criterios para admitir un Estado como nación: la existencia de un pasado, de una entidad histórica; de una lengua vernácula; y de la capacidad de conquista bélica.

Obviamente, estas consideraciones conceptuales del nacionalismo han ido modificándose a lo largo de los años, y en consecuencia, los criterios de constitución del nacionalismo. En este sentido, el deporte se ha convertido en el vehículo adoptado por los Estados para demostrar la conquista sobre el resto. Hobsbawm (1992) detalla al respecto que entre las dos guerras mundiales el deporte de espectáculo se convirtió en un combate entre gladiadores que simbolizaban Estados-nación; el deporte como lucha nacional. De hecho, Beck (2003) describe cómo la rivalidad británica-alemana tras la segunda guerra mundial se podía identificar a través de los acontecimientos futbolísticos; tendencia que se sigue reflejando tras el transcurso de las décadas.

Hobsbawm (1992) admite que el deporte es un instrumento eficaz para inculcar sentimientos nacionales, ya que, una selección nacional está encarnada por individuos concretos, normalmente conocidos y populares, los cuales, dominan un lenguaje universal (GUEDES, 2014) con el que cualquier persona se puede identificar. Es decir, cualquiera se siente capaz de golpear un balón, mientras que una ecuación de segundo grado resulta prácticamente inaccesible para la totalidad de la población. En esta misma línea, el valor atribuido a quienes practican el juego por su talento deportivo, así como, sus tradiciones invocadas a través de su individualidad, son componentes que permiten la construcción de la identidad nacional a través del fenómeno deportivo (GUEDES, 2014).

Los medios de comunicación utilizan deliberadamente estos espectáculos deportivos de masa como mecanismos de construcción cultural. Helal y Soares (2014) mantienen que la prensa deportiva ejerce una gran influencia en la construcción nacional de los Estados, confirmando y describiendo mitologías y discursos de identidad nacional. Por ejemplo, Jiang (2013) analizó la prensa de Hong Kong durante la celebración de los Juegos Olímpicos de verano de 2008 y 2012, en Pekín y Londres respectivamente. Todas las noticias se centraron en convertir a los campeones olímpicos en héroes nacionales, fomentando así el orgullo y la identidad nacional.

En consecuencia, los efectos propagandísticos de una medalla olímpica o la victoria de un campeonato internacional, suponen un elemento básico de cohesión de una sociedad (COLOMÉ, 2007, PAYERO, 2009). Las identidades grupales requieren una alimentación constante, de ahí que, el deporte se haya convertido en un excelente mecanismo de retroalimentación (CAPISTEGUI, 2012).

De esta manera, la utilización del deporte por parte del Estado se ha convertido en una buena fórmula para exhibir la nación, y la utilización del éxito deportivo ha sido y continúa siendo, una práctica habitual de los nacionalismos (GONZÁLEZ-RAMALLAL, 2008). Las manifestaciones e intereses nacionalistas encuentran en el deporte un adecuado cauce de expresión, y esto es debido a su aparente calidad de inofensivo por su carácter lúdico 
(PAYERO, 2009). Se puede considerar que el deporte y la política son un matrimonio perfecto que intenta demostrar la supremacía social, económica o política de una nación sobre otra (LIN; LEE; NAI, 2009).

El desarrollo deportivo se ha visto claramente favorecido por sus vínculos con las ambiciones del nacionalismo, ya sea expansionista o resistente, $y$, a pesar de las presiones de la globalización, sigue siendo un elemento persistente y robusto que muestra paradojas entre la globalización y la localización (BAIRNER, 2008). Un claro ejemplo se observa en Nakamura (2005) cuando analizó los discursos nacionalistas procedentes de los medios de comunicación sobre un jugador japonés en la liga profesional de béisbol de EEUU. El traspaso del jugador a la liga profesional es una evidencia de la globalización, mientras que, los discursos patrióticos a partir de su nacionalidad nipona son muestras de intereses locales vinculados con una nación concreta. Por lo tanto, un fenómeno aparentemente globalizador como es una liga profesional deportiva o unos Juegos Olímpicos, puede ejercer una influencia en la imagen internacional proyectada del país o de la ciudad, estableciéndose así, una interacción constate entre la identidad local y global (GARCÍA, 2009).

De hecho, algunos autores como Machado y Puig (2009) y García (2009) utilizan el término "glocalización" para referirse a esta ambigüedad del deporte ante lo global y lo local. Dicha tesitura es el reflejo de la crisis actual del Estado-nación, donde sus límites son porosos (GUEDES, 2014), provocando fenómenos de interdependencia económica y política, determinando por tanto, un nuevo proceso de búsqueda de identidades nuevas, ya sean locales o globales. Algunos autores como Hall (citado por GUEDES, 2014) aluden que esta situación provoca el ensalzamiento de los nacionalismos y fundamentalismos.

Son numerosas las investigaciones que constatan la utilización del deporte por parte del Estado con fines nacionalistas. Un claro ejemplo es el que exponen Viuda-Serrano y González (2012) cuando muestran el estrecho vínculo entre el fascismo y el franquismo y su utilización propagandística del deporte como medio de adoctrinamiento estatal. Más concretamente en España, Simón (2012) expone cómo el franquismo utilizó el enorme impacto del fútbol en la sociedad española para constituir nuevos héroes deportivos a partir de la industria cinematográfica, en este caso, encarnados en las figuras futbolísticas de Ladislao Kubala (jugador del Fútbol Club Barcelona) y Alfredo Di Stéfano (jugador del Real Madrid). Angelotti (2011) por su parte, demostró la utilización del deporte para la construcción de la identidad nacional en el periodo de post-revolución mexicana (1917-1940).

En las últimas décadas se ha comprobado la creciente importancia económica que genera la industria del deporte, reflejándose en el aumento de la promoción de las políticas públicas (PAWLOWSKI; DOWNWARD; RASCUITE, 2014). Incluso algunos autores recomiendan a los Estados invertir en el deporte de élite para proporcionar éxitos deportivos vinculados con las selecciones nacionales. De esta manera, se generan estados de bienestar subjetivos de la sociedad, afectando en las percepciones y expectativas sobre la situación económica del país (WICKER; PRINZ; HANAU, 2010).

En contraposición, Pawlowski, Downward y Rascuite (2014) no confirman que el orgullo constituido tras un éxito deportivo internacional contribuya al bienestar subjetivo de la sociedad. Sí mantienen que, la organización de eventos deportivos internacionales, como un campeonato del mundo o unos Juegos Olímpicos, resultan más trascendentales que el éxito deportivo en sí. 
En esta misma línea, García (2009) constató que los Juegos Olímpicos de Barcelona en 1992 sirvieron para reconciliar el antagonismo entre España y Cataluña, ya que, el notable éxito organizativo, la gran actuación del equipo español, así como la ausencia de conflictos durante la celebración, contribuyeron a que el evento fuese percibido muy positivamente desde Cataluña y el resto de España.

Gleaves y Llewellyn (2014) son muy críticos cuando cuestionan la necesaria vinculación entre el deporte de élite y el nacionalismo. De hecho, ponen en duda los aspectos positivos que provienen de dicha connivencia, resaltando los dilemas éticos por la discordia internacional que ocasionan estos eventos deportivos. Plantean que la desmembración del deporte de élitenación no generaría inferiores niveles de competencia deportiva, es decir, las diferencias nacionales deportivas no son un agente de estimulación de la eficacia y competencia deportiva. Por lo tanto, consideran que las identidades nacionales tendrían que tener un papel mucho más reducido, incluso ninguno, en el deporte de élite.

Los partidos políticos no son ajenos a la trascendencia y relevancia socioeconómica del deporte en general, y más específicamente, sobre el deporte de élite. La presencia del deporte en las políticas estatales ha sido estudiada en los últimos años (AUGÉ, 2011, HOULIHAM, 2012, LIN; LEE; NAI, 2009, PUIG; MARTÍNEZ; GARCÍA, 2010), centrando principalmente su análisis en las políticas implementadas por los gobiernos en curso, y no así, en las propuestas electorales de los partidos políticos.

Algunos de los resultados de estas investigaciones muestran mensajes contradictorios y paradójicos por la multiplicidad de factores, pero que engloban una retórica común de eficiencia, competitividad y liderazgo (GREEN, 2006, PIGGIN; JACKSON; MALCOM, 2009). Algunas otras, como la investigación realizada por Augé (2011) sobre la transición democrática española (1977-1988) de los partidos políticos catalanes, reveló el pluralismo ideológico de los partidos, hecho que no puede ser constatado en el presente actual (BERGSGARD et al., 2007).

Este artículo pretende comprobar, por medio del análisis de contenido de los programas electorales, si el deporte es utilizado por los partidos políticos españoles como mecanismo de identidad cultural y vida nacional. Estos resultados forman parte del proyecto POLCOR ("Concepciones del cuerpo y cultura corporal en las propuestas de actividad física y deporte de los programas políticos electorales de las elecciones generales de 2011 en España"), con código PMAFI/07/12, evaluado positivamente por la por la Agencia Nacional de Evaluación y Prospectiva (ANEP) del Gobierno de España y financiado por la Fundación Universitaria San Antonio de Murcia (España).

La hipótesis de partida es: los partidos políticos en España utilizan el deporte en su discurso programático como estrategia para reforzar la identidad cultural de un territorio y patrocinar la nación.

\section{METODOLOGÍA}

\subsection{Técnica de análisis}

Se utilizó el análisis de contenido como técnica de investigación, ya que, permite examinar y explicar objetiva, sistemática, cuantitativa y cualitativamente las formas y los 
significados de los mensajes (KRIPPENDORFF, 1990). Se diseñó un sistema de categorías para analizar los discursos políticos. Este sistema de categorías se centró en el nivel semántico, combinado con una estrategia extensiva (NAVARRO; DÍAZ, 1994), ya que, el análisis debía de ser temático.

Los resultados del presente artículo han sido extraídos de un sistema de categorías elaborado a partir del proyecto sobre políticas corporales POLCOR, el cual, tiene cuatro dimensiones de análisis: cultura corporal; políticas corporales; sistema de organización y gestión; y atención a la diversidad. Dichos resultados pertenecen a la subcategoría vida nacional, incluida en la dimensión política corporal y la categoría prioridades políticas sobre cultura corporal. En el Quadro 1 se puede consultar la definición de la dimensión, categoría y subcategoría utilizadas para este artículo.

Quadro 1 - Definición de la dimensión, categoría y subcategoría

\begin{tabular}{|c|c|c|}
\hline $\begin{array}{l}\text { Dimensión políticas } \\
\text { corporales }\end{array}$ & $\begin{array}{l}\text { Categoría prioridades políticas } \\
\text { sobre cultura corporal }\end{array}$ & Subcategoría vida nacional \\
\hline $\begin{array}{l}\text { En esta dimensión se recogen } \\
\text { todos aquellos enunciados que } \\
\text { se refieren a las potencialidades } \\
\text { que los partidos políticos otorgan } \\
\text { a la cultura corporal vinculada } \\
\text { al campo de las actividades } \\
\text { físicas deportivas; así como a las } \\
\text { prioridades políticas y formas de } \\
\text { actuación respecto a la cultura } \\
\text { corporal que se derivan de estos } \\
\text { posicionamientos ideológicos. }\end{array}$ & $\begin{array}{l}\text { (Adaptada de Augé, 2011). } \\
\text { Objetivos que cada partido } \\
\text { se plantea alcanzar mediante } \\
\text { actuaciones relacionadas con la } \\
\text { cultura corporal vinculada a las } \\
\text { actividades físicas deportivas } \\
\text { y propuestas de actuación } \\
\text { concretas que se presentan }\end{array}$ & $\begin{array}{l}\text { (Adaptado de Werner; Lacewell; } \\
\text { Volkens, 2010). } \\
\text { Referencias favorables a la protección } \\
\text { de la nación del país o comunidad } \\
\text { autónoma del programa electoral a } \\
\text { través de las expresiones motrices. } \\
\text { Puede incluir: apoyo a la creación y/o } \\
\text { defensa de selecciones, federaciones, } \\
\text { clubes o competiciones deportivas } \\
\text { nacionales; llamamientos generales al } \\
\text { patriotismo y/o nacionalismo como eje } \\
\text { de los proyectos deportivos; protección } \\
\text { y promoción de la lengua vernácula del } \\
\text { territorio a través de las expresiones } \\
\text { motrices. }\end{array}$ \\
\hline
\end{tabular}

Fuente: elaboración propia

\subsection{Muestra}

La población del estudio -universo- estuvo formada por el conjunto de programas electorales de los partidos políticos que presentaron candidatura a las elecciones generales del 2011 en España para el Congreso de los Diputados y al Senado convocadas por Real Decreto 1329/2011, de 26 de septiembre, candidaturas que fueron publicadas en el Boletín Oficial del Estado número 252, de 19 de octubre de 2011.

De los 118 partidos que presentaron candidatura se tuvo acceso a un total de 94 programas, de los cuales, 38 eran partidos de coalición que proponían programas idénticos. Por lo tanto, se analizaron 56 programas electorales, siendo ocho de ellos los que contienen referencias que sitúan al deporte como medio para impulsar el patriotismo.

Es decir, la muestra está compuesta por ocho programas electorales de los siguientes partidos políticos: Bloque nacionalista galego (BNG); Convergencia i unió (CiU); Bloc-iniciativa verds (EQUO); Coalició compromís (compromiso Q); Esquerra republicana balear (Esquerra); 
Foro Asturias ciudadanos (FAC); Falange española de las Jons (FE de las Jons); Partido popular (PP) y Partido socialista obrero español (PSOE).

\subsection{Procedimiento}

El procedimiento se organizó en torno a tres fases principales, que coinciden con las propuestas por Bardin (2002): 1. Preanálisis, 2. Explotación del material, 3. Tratamiento e interpretación de los resultados.

En la fase de preanálisis se confeccionó el sistema de categorías para el proyecto POLCOR. Para llevarlo a cabo, resultó imposible traspasar ciertos modelos de categorías estructuradas ya confeccionados para el análisis de las políticas deportivas por estar contextualizados en otros países, o por no incorporar todos los matices del objeto de estudio, por lo que fue necesario confeccionar un sistema de codificación elaborado ad hoc.

La elaboración se inició con la consecución de categorías e indicadores derivados deductivamente a partir de los conceptos teóricos y los estudios previos, y se complementó mediante un proceso inductivo o empírico, resultado de la lectura superficial de los programas. Este primer contacto con los documentos de análisis fue realizado por cuatro investigadoras que participaban en el proyecto. Una vez establecida una serie provisional de dimensiones, categorías y subcategorías, éstas fueron examinadas, criticadas y modificadas por todo el equipo.

En el preanálisis se llevaron a cabo una serie de controles: prueba piloto, consulta a expertos y pretest de fiabilidad.

En la prueba piloto se codificó una pequeña muestra de textos con una primera versión de codificación. Las personas que participaron en el proyecto codificaron una submuestra de textos elegidos al azar. Realizada esta tarea de forma individual se discutieron en grupo las soluciones. El resultado fue una reformulación e incorporación de nuevas categorías y la confirmación de su eficacia y pertinencia.

Esta segunda versión fue examinada y evaluada por tres expertos/as. Las tres personas expertas evaluaron la claridad, exhaustividad, exclusión mutua de las subcategorías, homogeneidad, pertinencia, objetividad y productividad del manual de codificación. Estos ítems fueron calificados con valores de 4 ó 5 , en una escala de Likert 1-5. Los comentarios cualitativos reforzaron la propuesta.

El pretest de fiabilidad consistió en un cálculo de acuerdo entre codificadores independientes. Se codificaron los discursos del $20 \%$ de los programas. Se manejó una submuestra aleatoria y otra submuestra de fragmentos seleccionados intencionalmente de tal forma que posibilitaran testar el máximo de categorías (NEUENDORF, 2009). Se utilizó el coeficiente kappa de Cohen y la alpha de Krippendorff como medidas de fiabilidad, siendo satisfactoria en ambas ( $k=.906 ; \alpha=.8559)$.

Durante la explotación del material se realizó una lectura íntegra de los programas electorales y la descomposición de los mismos en unidades de registro. Todas las unidades de registro fueron codificadas de forma consensuada por las investigadoras del proyecto. Una de las investigadoras participó en la codificación colectiva de un número pequeño de textos de muestra. Después de la formación presencial, esta persona codificó una submuestra del corpus de forma individual para la realización de un nuevo test de fiabilidad. 
Finalizada la codificación se realizó una nueva prueba de fiabilidad intercodificadores para aumentar la consistencia de los resultados obtenidos, mostrando confianza tanto con la kappa de Cohen ( $k=.872)$ como con la alpha de Krippendorff $(\alpha=.8726)$.

La última fase, el tratamiento e interpretación de los resultados se realizó desde un enfoque cuantitativo, utilizando el paquete estadístico SPSS 20; y fue complementado mediante el análisis de los discursos, manejando el programa para el análisis de datos cualitativos NVivo 10.

\section{RESULTADOS}

De los 27 programas que recogen referencias a las prácticas físico-deportivas en sus programas electorales, ocho (29.6\%) contienen referencias que sitúan al deporte como medio para impulsar el patriotismo. Estos partidos son: BNG, CiU, Compromis Q Esquerra, FAC, FE de las Jons, PP, y PSOE. El gráfico 1 detalla el número de referencias por partido.

Gráfico 1 - Número de referencias por partido político

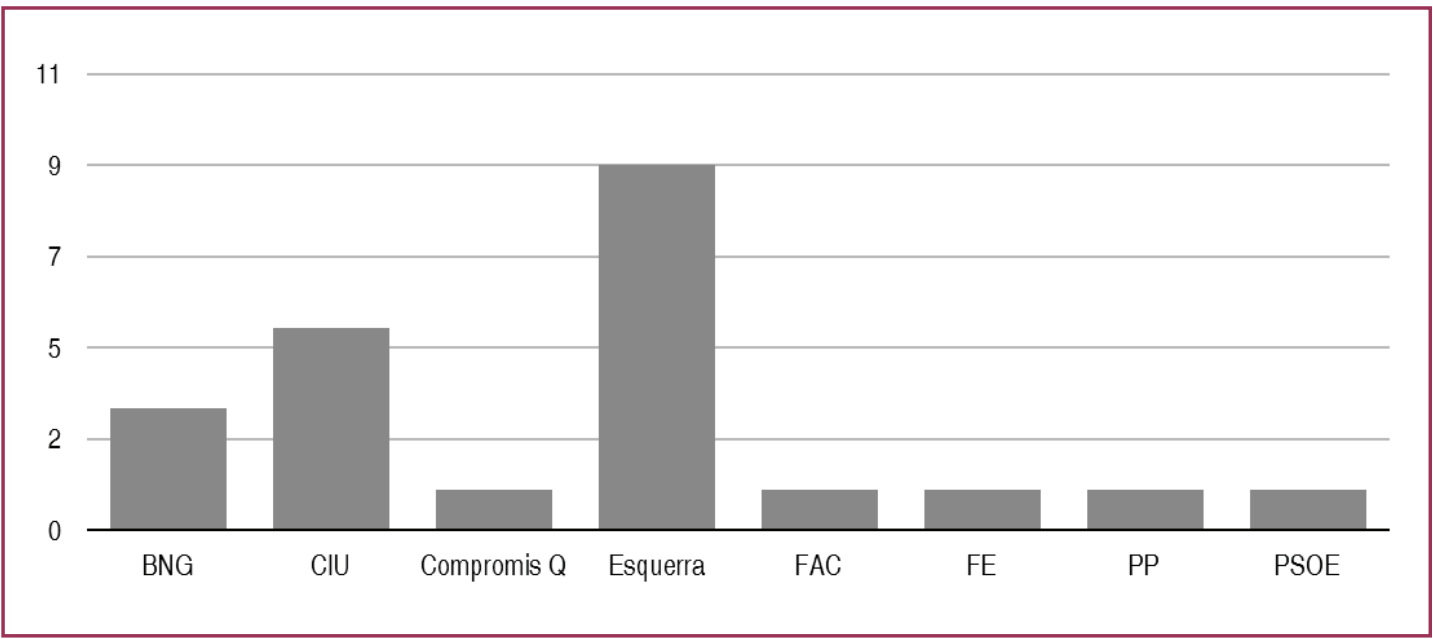

Fuente: elaboración propia

Los tres partidos con mayor número de referencias son partidos nacionalistas, es decir, tienen una clara tendencia política encaminada hacia la independencia de la Comunidad Autónoma ${ }^{1}$ en una nación independiente y soberana. Esquerra y CiU son partidos nacionalistas catalanes ${ }^{2}$, mientras que el BNG es un partido nacionalista gallego.

La creación y/o defensa de selecciones, federaciones, clubes y/o competiciones deportivas propias es una de las estrategias más utilizadas por estos partidos nacionalistas. Proponen la constitución de selecciones autonómicas para poder participar en competiciones internacionales, es decir, la selección deportiva es la que permite situar al territorio como nación en el escenario internacional. Por ejemplo, el BNG propone "Articular a participación das seleccións deportivas galegas en competicións internacionais e a representación das

\footnotetext{
1 España se organiza territorial y administrativamente en 17 comunidades autónomas (además de en 2 ciudades autónomas): Andalucía, Aragón, Canarias, Castilla y León, Castilla La Mancha, Cantabria, Cataluña, Comunidad Foral de Navarra, Comunidad Valenciana, Comunidad de Murcia, Comunidad de Madrid, Extremadura, Galicia, Islas Baleares, La Rioja, País Vasco y Principado de Asturias (Ceuta y Melilla). 
federacións deportivas galegas nos organismos deportivos internacionais de xeito directo"3 ( $p$. 35). Por su parte, el epígrafe del programa electoral de Esquerra sobre política deportiva es muy preciso: "Esports: una nació, una selecció"4 (p.120).

Para ello, solicitan la posibilidad de desarrollar marcos organizativos propios, independientes del Estado español, tal y como pueda ser la constitución del comité olímpico catalán "Instarem el Govern de l'Estat a fer les gestions que siguin necessàries per eliminar els obstacles que a nivell internacional dificulten la creació del Comitè Olímpic de Catalunya" (CiU, p.78); o "la integración directa de les federacions esportives catalanes, com a membres de le dret, a les federacions internacionals cirresponents"5 (CiU, en el apartado 3.1.2. Esports).

Incluso, Esquerra propone modificar la normativa española para que las federaciones deportivas catalanas, vascas, gallegas y de otros territorios del Estado, puedan afiliarse a otras entidades de ámbito español, europeo o mundial. El mismo programa solicita la libertad de los deportistas (no sólo catalanes, también vascos, gallegos y de todo el territorio español) para escoger entre la selección estatal o autonómica con la que participar en las competiciones internacionales oficiales (p. 120).

El deporte también es utilizado por estos partidos como mecanismo de protección y promoción de la lengua vernácula del territorio. El BNG se compromete en su programa electoral a la difusión y promoción del gallego, ampliando las bandas horarias en las que se retransmitan los acontecimientos deportivos en gallego (p. 32). Por su parte, CiU establece el fomento del catalán en el deporte (apartado 3.1.2. Esports). Otros partidos que no son nacionalistas, como el PP6 (p. 203), el PSOE “ (p. 91), y la FE de las Jons (p. 18), sitúan al deporte, junto con otras acciones de la sociedad, como promotoras de la difusión internacional del español.

El resto de los partidos políticos también utilizan el deporte en sus discursos para fortalecer el nacionalismo, pero en estos casos defienden un nacionalismo español. Por ejemplo, el FAC dice que el deporte es un elemento aglutinador de la sociedad española, en la que todos los ciudadanos se sienten partícipes de un proyecto común (p. 81).

Pero sin duda alguna, la idea del deporte como proyección internacional es la idea más abordada por los partidos políticos, sean de corte nacionalista o no. Es decir, las selecciones deportivas que representan a una nación en competiciones internacionales, son un adecuado escenario propagandístico internacional, es decir, colocan a la nación representada ante un reconocimiento mundial. Por ejemplo, el PP expone que la acción deportiva será "una guía en nuestra acción exterior" (p. 203), el PSOE7 matiza que "el desarrollo del deporte profesional sólo tiene sentido desde lo público como elemento de proyección internacional de España" ( $p$. 91), y la FE de las Jons establece que el deporte es un mecanismo de difusión de la lengua y de la cultura española (p. 18).

3 "Articular la participación de las selecciones deportivas gallegas en competiciones internacionales y la representación de las federaciones deportivas gallegas en los organismos deportivos internacionales de forma directa" (N. del A.).

4 "Deportes: una nación, una selección" (N. del A.).

5 "[...] la integración directa de las federaciones deportivas catalanas, como miembros de pleno derecho, a las federaciones internacionales correspondientes" (N. del A.).

6 El Partido Popular ganó las elecciones generales de 2011 logrando la mayoría absoluta, con un total de 10.830 .693 votos y 186 escaños en el Congreso de los Diputados.

7 EI PSOE fue el segundo partido más votado en las últimas elecciones generales, consiguiendo un total de 6.973 .880 votos y 110 escaños. 
Por último, Compromis $\mathrm{Q}^{8}$ difiere del resto de propuestas electorales, ya que, detalla en su página 213: "Exigir la domiciliación fiscal en territorio español para poder representar a España en cualquier actividad deportiva".

El gráfico 2 muestra el porcentaje de la muestra según los contenidos identificados en sus propuestas electorales, es decir, nos permite exponer los intereses más requeridos por los partidos políticos. Se puede comprobar cómo el $62,5 \%$ de la muestra utiliza el deporte como mecanismo de proyección internacional (PSOE, CiU, FE de las Jons, Esquerra y PP). El 50\% (BNG, CiU, PP y FE de las Jons) se sirve del deporte para la difusión o defensa de la lengua. El 37,5\%, que son todos los partidos nacionalistas de la muestra (BNG, CiU y Esquerra), tienen una explícita tendencia independentista en sus discursos deportivos. De forma más minoritaria, la cohesión social y la domiciliación fiscal son defendidos por el 12,5\% (FAC y Compromis Q respectivamente).

Gráfico 2 - Porcentaje de la muestra por contenidos

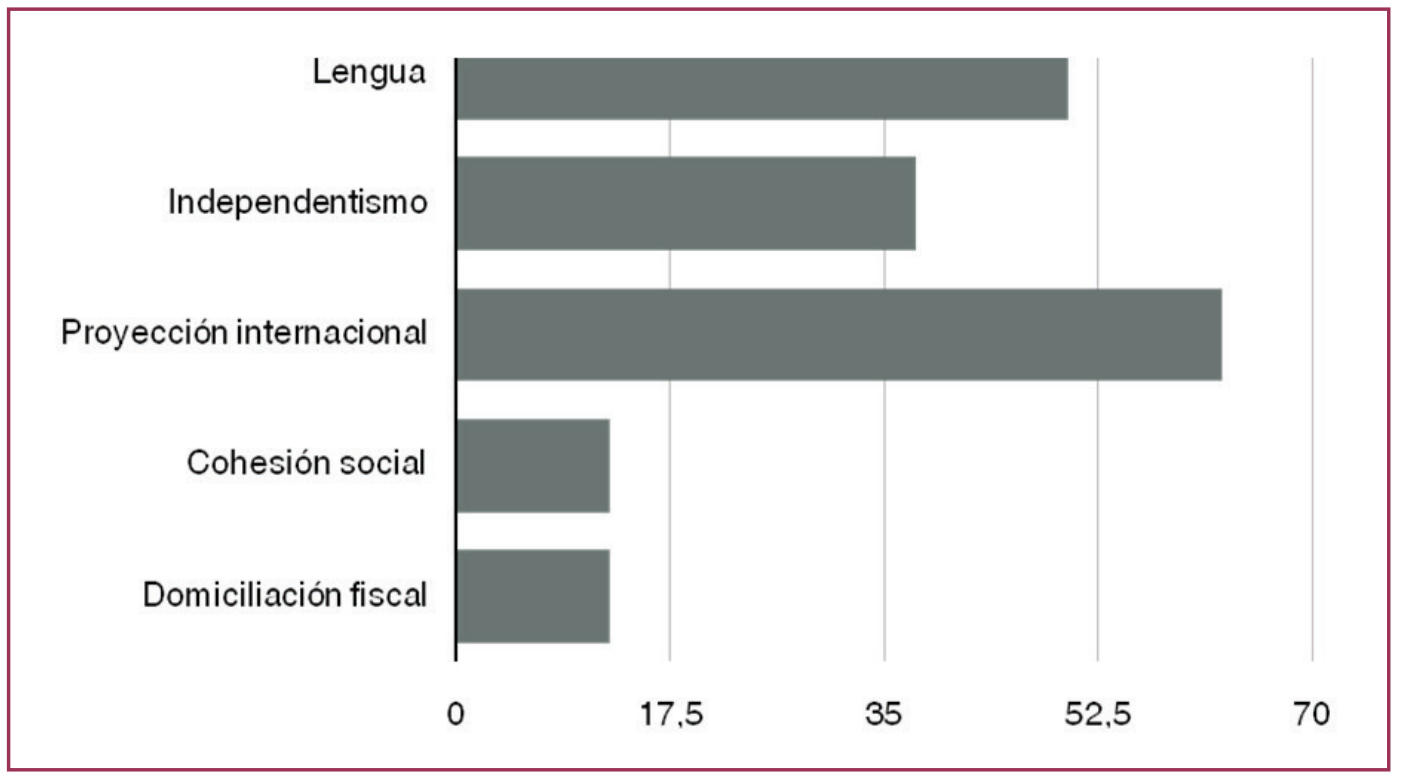

Fuente: elaboración propia

\section{DISCUSIÓN}

Los resultados de esta investigación revelan que los programas electorales de algunos de los partidos políticos que se presentaron en las elecciones generales de 2011 utilizan el deporte como mecanismo de identidad cultural de un territorio y patrocinan el nacionalismo. Es decir, esta investigación confirma la tesis de Lin, Lee y Nai (2009) en cuanto que el deporte y la política son un matrimonio perfecto que intenta demostrar la supremacía de una nación sobre otra.

Sobre todo, son los partidos políticos nacionalistas los que más desarrollan los discursos independentistas en torno al deporte, sobre todo, referido al deporte de élite. Es decir, solicitan que las selecciones autonómicas deportivas puedan participar en competiciones internacionales, ya que de esta manera, se coloca el territorio autonómico al mismo nivel que el resto de naciones. Estos resultados constatan las tesis de Hobsbawm (1992) en cuanto que el deporte es un instrumento eficaz para inculcar sentimientos nacionales debido a que las 
selecciones nacionales deportivas están encarnadas por individuos concretos, en los cuales, cualquier ciudadano se puede identificar (GUEDES, 2014). Paralelamente, estos resultados verifican las investigaciones de carácter histórico que demuestran la utilización del deporte por parte del Estado con fines de adoctrinamiento nacionalista (ANGELOTTI, 2011, SIMÓN, 2012, VIUDA-SERRANO; GONZÁLEZ, 2012).

Los resultados de la presente investigación relacionados con el uso del deporte como proyección internacional confirman los resultados de González-Ramallal (2008) referidos a la utilización del deporte por parte del Estado para exhibir la nación. También permite corroborar la proyección internacional que suponen los eventos deportivos internacionales, los cuales, son simbolizados como combates entre naciones (BECK, 2003, HOBSBAW, 1992). En la misma medida, ratifica los resultados de las investigaciones centradas en los medios de comunicación que describen la proyección internacional a través de los discursos mitológicos sobre las selecciones deportivas nacionales y/o sus héroes deportivos (HELAL; SOARES, 2014, JIANG, 2013, NAKAMURA, 2005).

Relacionado con la proyección internacional, la promoción y/o protección de la lengua vernácula desvelada en las propuestas políticas deportivas de los partidos políticos de la muestra del presente estudio, muestra concordancia con lo establecido por Hobsbawm (1992) en cuanto que la lengua era uno de los criterios para admitir un Estado como nación.

También esta investigación permite aseverar la cohesión social como contenido de utilización deliberada a partir de un éxito deportivo (COLOMÉ 2007, PAYERO, 2009), suponiendo en consecuencia, una retroalimentación constante de identidad grupal (CAPISTEGUI, 2012).

La característica lúdica del deporte que alude Payero (2009), siendo ésta las que le convierte en un excelente cauce de expresión nacionalista, no puede ser constatada por la presente investigación, ya que, las atribuciones al deporte con fines recreativos, de entretenimiento y diversión, están incluidas en otra subcategoría de análisis -2.2.2. Recreación-, la cual, no es objeto de análisis para este estudio.

Esta investigación ha permitido evidenciar la notable paradoja entre la globalidad y la localidad (BAIRNER, 2008) que tiene el discurso deportivo, lo que algunos autores han denominado como "glocalización" (GARCÍA, 2009, MACHADO; PUIG, 2009). Las promesas electorales de los partidos políticos de la muestra utilizan esta ambigüedad conceptual, ya que, utilizan los beneficios globales del sistema deportivo para sus intereses independentistas locales.

A pesar de que el diseño de la presente investigación no permite corroborar los resultados de Pawlowski, Downward y Rasciute (2014) vinculados con el aumento de la promoción de las políticas públicas debido a la creciente importancia económica de la industria del deporte, sí ha permitido constatar la promoción pública del deporte con finalidad de exaltación nacional en las promesas electorales de los partidos políticos de la muestra.

Los dos partidos políticos nacionalistas catalanes de la muestra, CiU y Esquerra, utilizan discursos políticos antagónicos hacia el Estado español, por lo que este resultado, no permite constatar lo que García (2009) desveló en cuanto que los Juegos Olímpicos de Barcelona de 1992 sirvieron para reconciliar las diferencias entre Cataluña y España. De todas maneras, esta investigación refleja únicamente el contenido de los programas electorales de los partidos 
políticos, quedándose al margen las opiniones o el imaginario de la sociedad española frente al fenómeno deportivo.

\section{CONCLUSIONES}

Este tipo de investigaciones sociales permiten comprender el tipo de racionalidad que utiliza el deporte como mecanismo de control y normalización de la población. Más concretamente, este artículo ha constatado que los partidos políticos españoles de la muestra utilizan el deporte en sus discursos de los programas electorales con fines propagandísticos nacionalistas, es decir, como estrategia para reforzar la identidad nacional, ya sea autonómica o estatal. Sobre todo, son los partidos nacionalistas los que mayor discurso político deportivo utilizan con esta finalidad, circunscribiéndolo específicamente a intereses independentistas.

Los contenidos más usados en los discursos políticos de las promesas electorales de los partidos de la muestra son: la protección y/o promoción de la lengua vernácula; el independentismo -sólo los partidos nacionalistas-; y la proyección internacional.

\section{REFERENCIAS}

ANGELOTTI, Gabriel. Deporte y nacionalismo en México durante la post revolución. Revista de História do Esporte, Rio de Janeiro, v. 4, n. 1, p. 22-34, 2011.

AUGÉ, Aleix. El pluralismo ideológico en las propuestas de política deportiva en los inicios de la democracia española. Estudio del caso de Cataluña (1977-1988). Materiales para la Historia del Deporte, Barcelona, v. 9, p. 103-119, 2011.

BAIRNER, Alan. Sport, nationalism and globalization: relevance, impact, consequences. Hitotsubashi journal of arts and sciences, Tokio, v. 49, n. 1, p. 43-53, 2008.

BARDIN, Laurence. Análisis de contenido. Madrid: Akal, 2002.

BECK, Peter. The relevance of the 'irrelevant': Football as a missing dimension in the study of British relations with Germany. International Affaires, Hoboken, v. 79, n. 2, p. 389-411, 2003.

BERSGARD, Nils Asle et al. Sport policy: a comparative analysis of stability and change. Oxford: Elsevier, 2007.

BLOQUE NACIONALISTA GALEGO. Programa electoral eleccións xerais 2011. Disponible en: < http://politica.elpais.com/especiales/2011/elecciones_20n/documentos/programa_bng.pdf>. Acceso en: 9 nov. 2013.

CAPISTEGUI, Francisco Javier. Deporte e identidad, o sobre cómo definirnos. Historia y Comunicación Social, Madrid, v. 17, p. 19-39, 2012.

COALICIÓ COMPROMIS Q. Programa elecciones generales 2011. Disponible en: <http://joanbaldovi. com/programa/Programa_Compromis-Q_ES.pdf>. Acceso en: 23 nov. 2013.

COLOMÉ, Gabriel. Una nota sobre deporte y política. In: TAIBO, Carlos. (Ed.). Nacionalismo español: esencias, memorias e instituciones. Madrid: Catarata, 2007. p. 377-383. 
CONVERGĖNCIA I UNIÓ. Programa electoral de Convergéncia i Unió. Eleccions generals novembre 2011. Disponible en: <http://www.elmundo.es/elecciones/elecciones-generales/2011/programas/ciu. html>. Acceso en: 9 nov. 2013.

ESQUERRA REPUBLICANA DE CATALUNYA. Eleccions a les corts espanyoles 2011, programa electoral. Disponible en: <http://www.esquerra.cat/partit/programes/e2011_programa.pdf> Acceso en: 9 nov. 2013.

FALANGE ESPAÑOLA DE LAS JONS. Programa electoral 2011. Disponible en: <http://bit.ly/ uhgUoE.> Acceso en: 3 nov. 2013.

FORO ASTURIAS CIUDADANOS. Mas foro, mejor España. Elecciones generales 2011. Disponible en: <http://www.foroasturias.es/programa-electoral-20-noviembre/>. Acceso en: 3 oct. 2013.

GARCÍA, Manuel. El deporte rompe la tendencia globalizadora, se fundamenta en lo local (glocalización). Apunts. Educación física y deportes, Barcelona, v. 97, p. 29-36, 2009.

GLEAVES, John; LLEWELLYN, Matthew. Ethics, nationalism, and the imagined community: the case against international sport. Journal of Philosophy of Sport, Boston, v. 41, n. 1, p. 1-19, 2014.

GONZÁLEZ-RAMALLAL, Manuel Eduardo. La identidad contada: la información deportiva en torno a la selección española de fútbol. Universitas Humanística, Bogotá, v. 66, p. 219-238, 2008.

GREEN, Mick. From 'Sport for All' to Not About 'Sport? at All?: Interrogating Sport Policy Interventions in the United Kingdom. European Sport Management Quarterly, London, v. 6, n. 3, p. 217-238, 2006.

GUEDES, Simone Lahud. On criollos and capoeiras: notes on soccer and national identity in Argentina and in Brazil. Soccer and Society, London, v. 15, n. 1, p. 147-161, 2014.

HOBSBAW, Eric. Naciones y nacionalismo desde 1870. Barcelona: Crítica, 1992.

HOULIHAM, Barrie. Sport polcy convergence: a framework for analysis. European Sport Management Quarterly, Oxforshire, v. 12, n. 2, p. 111-135, 2012.

HELAL, R.; SOARES, A. J. The decline of the 'Soccer-Nation': Journalism, soccer and national identity in the 2002 World Cup. Soccer and Society, London, v. 15, n. 1, p. 132-146, 2014.

JIANG, Qiaolei. Celebrity athletes, soft power and national identity: Hong Kong newspaper coverage of the olympic champions of Beijing 2008 and London 2012. Mass Communication and Society, Oxforshire, v. 16, n. 6, p. 888-909, 2013.

KRIPPENDORFF, Klaus. Metodología de análisis de contenido: teoría y práctica. Paidós: Barcelona, 1990.

LIN, Chien-Yu; LEE, Ping-Chao; NAI, Hui-Frang. Theorizing the role of sport in state-politics. International Journal of Sport and Exercise Science, Taiwan, v. 1, n. 1, p. 23-32, 2009.

MACHADO, Rui; PUIG, Nuria. El deporte, entre lo local y lo global: ¿una mirada europea? Apunts: Educación física y deportes, Barcelona, v. 97, p. 3-6, 2009.

NAKAMURA, Yuka. The samurai sword cuts both ways. A transnational analysis of japanese and US media representations of Ichiro. International Review for the Sociology of Sport, London, v. 40, n. 4, p. 467-480, 2005. 
NAVARRO, Pablo; DIAZ Capitalina. Análisis de contenido. In: DELGADO, José Manuel; GUTIÉRREZ, Juan (Eds.); Métodos y técnicas cualitativas de investigación en ciencias sociales. Madrid: Síntesis, 1994. p. 23-43.

NEUENDORF, Kimberly A. Reliability for content analysis. In JORDAN, A. B.; KUNKEL, J.; MANGANELLO Y FISBBEIN (Eds.). Media messages and public health: a decisions approach to content analysis. New York: Routledge, 2009. p. 67-87.

PARTIDO POPULAR. Programa electoral Partido Popular. Más sociedad, mejor gobierno. Disponible en: < http://www.pp.es/sites/default/files/documentos/5751-20111101123811.pdf>. Acceso en: 7 oct. 2013.

PARTIDO SOCIALISTA OBRERO ESPAÑOL. Programa electoral elecciones generales 2011.

Disponible en: <https://www.psoe.es/source-media/0000000493500/000000493991.pdf>. Acceso en: 5 nov. 2013.

PAWLOWSKI, Tim; DOWNWARD, Paul; RASCIUTE, Simona. Does national pride from international sporting success contribute to well-being? An international investigation. Sport Management Review, Auckland, v. 17, p. 121-132, 2014.

PAYERO, Lucía. La nación se la juega: relaciones entre el nacionalismo y el deporte en España. Agora para la educación física y el deporte, Valladolid, v. 10, p. 81-118, 2009.

PIGGIN, Joe; JACKSON, Steven; MALCOLM, Lewis. Telling the truth in public policy: an analysis of New Zealand sport policy discourse. Sociology of Sport Journal, Champaing, v. 26, p. 462-482, 2009.

PUIG, Nuria; MARTÍNEZ, Joaquin; GARCÍA, Borja. Sport policy in Spain. International Journal of Sport Policy and Politics, London, v. 2, n. 3, p. 381-390, 2010.

SIMÓN, Juan Antonio. Fútbol y cine en el franquismo: la utilización política del héroe deportivo en la España de Franco. Historia y Comunicación Social, Madrid, v. 17, p. 69-84, 2012.

VIUDA-SERRANO, Alejandro; GONZÁLEZ, Teresa. Héroes de papel: El deporte y la prensa como herramientas de propaganda política del fascismo y el franquismo. Historia y Comunicación Social, Madrid, v. 17, p. 41-68, 2012.

WERNER, Annika; LACEWELL, Onawa; VOLKENS, Andrea. Manifiesto Coding Instructions (en línea). Disponible en: https://manifesto-project.wzb.eu. Acceso en: 7 mayo 2014.

WICKER, Pamela; PRINZ, Joachim; HANAU, Tassilo van. Estimating the value of national sporting success. Sport Management Review, Auckland, v. 15, p. 200-210, 2010.

Financiación: Este trabajo se realizó dentro del proyecto de investigación "Concepciones del cuerpo y cultura corporal en las propuestas de actividad física y deporte de los programas políticos electorales de las elecciones generales del 2011 en España (POLCOR)", con código PMAFI/07/12, evaluado positivamente por la por la Agencia Nacional de Evaluación y Prospectiva (ANEP) del Gobierno de España y financiado por el Plan Propio de ayudas a la investigación -Convocatoria de ayudas a la realización de proyectos de investigación para 2012- de la Fundación Universitaria San Antonio de Murcia. 